\title{
SOLUTIONS DIFFERENTIABILITY OF PARAMETRIC OPTIMAL CONTROL FOR ELLIPTIC EQUATIONS
}

\author{
Kazimierz Malanowski \\ Systems Research Institute \\ Polish Academy of Sciences \\ ul. Newelska 6, 01-447 Warszawa, Poland \\ kmalan@ibspan.waw.pl
}

Abstract A family of parameter dependent elliptic optimal control problems with nonlinear boundary control is considered. The control function is subject to amplitude constraints. It is shown that under standard coercivity conditions the solutions to the problems are Bouligand differentiable (in $\left.L^{s}, s<\infty\right)$ functions of the parameter. The differentials are characterized as the solutions of accessory linear-quadratic problems.

Keywords: Parametric optimal control, elliptic equation, nonlinear boundary control, control constraints, Bouligand differentiability of the solutions

\section{Introduction}

In this paper, we analyse differentiability, with respect to the parameter, of solutions to a nonlinear boundary optimal control problem for an elliptic equation. Our aim is to show that, under a standard coercivity condition, the solutions to the optimal control problem are Bouligand differentiable functions of the parameter. Let us recall this concept of differentiability (see $[3,8,9]$ ).

Definition 1 A function $\phi$, from an open set $\mathcal{G}$ of a normed linear space $H$ into another normed linear space $X$, is called Bouligand differentiable (or B-differentiable) at a point $h_{0} \in \mathcal{G}$ if there exists a positively homogeneous mapping $D_{h} \phi\left(h_{0}\right): \mathcal{G} \rightarrow X$, called B-derivative, such that

$$
\phi\left(h_{0}+\Delta h\right)=\phi\left(h_{0}\right)+D_{h} \phi\left(h_{0}\right) \Delta h+o\left(\|\Delta h\|_{H}\right) .
$$

Clearly, if $D_{h} \phi\left(h_{0}\right)$ is linear, it becomes Fréchet derivative. 
As in [4] and in [5], the sensitivity, i.e., differentiability analysis for the original nonlinear problem is reduced to the same analysis for the accessory linear-quadratic problem. The starting point of the analysis is the Lipschitz stability result for the solutions to the linear-quadratic elliptic problems due to A.Unger [11]. Using this result, B-differentiability is proved in two steps. First, passing to the limit in the difference quotient, we show the directional differentiability and characterize the directional differential as the solution of an auxiliary linear-quadratic optimal control problem. Using this characterization, in the second step we show that an estimate of the form (1) holds, i.e., the solutions are Bouligand differentiable. This result can be considered as a generalization of that obtained in [1], where a different methodology was used to prove the directional differentiability of the solutions to parametric elliptic problem, under the assumption that the cost functional is quadratic with respect to the control.

\section{Preliminaries}

Let $\Omega \subset \mathbb{R}^{n}$ denote a bounded domain with boundary $\Gamma$. As usually, by $\Delta y$ and $\partial_{\nu} y$ we denote the Laplace operator and the co-normal derivative of $y$ at $\Gamma$, respectively. Moreover, let $H$ be a Banach space of parameters and $G \subset H$ an open and bounded set of feasible parameters.

For any $h \in G$ consider the following elliptic optimal control problem:

$$
\begin{aligned}
\left(\mathrm{O}_{h}\right) \quad \text { Find }\left(y_{h}, u_{h}\right) & \in Z^{\infty}:=\left(W^{1,2}(\Omega) \cap C(\bar{\Omega})\right) \times L^{\infty}(\Gamma) \text { such that } \\
F\left(y_{h}, u_{h}, h\right) & =\min \{F(y, u, h) \\
& \left.:=\int_{\Omega} \varphi(y(x), h) d x+\int_{\Gamma} \psi(y(x), u(x), h) d S_{x}\right\}
\end{aligned}
$$

subject to

$$
\begin{aligned}
& -\Delta y(x)+y(x)=0 \quad \text { in } \Omega, \\
& \partial_{\nu} y(x)=b(y(x), u(x), h) \quad \text { on } \Gamma, \\
& u \in \mathcal{U}:=\left\{v \in L^{\infty}(\Gamma) \mid m_{1} \leq v(x) \leq m_{2} \text { a.e. in } \Gamma\right\} \text {. }
\end{aligned}
$$

In this setting, $m_{1}<m_{2}$ are fixed real numbers, $d S_{x}$ denotes the surface measure induced on $\Gamma$, and the subscript $x$ indicates that the integration is performed with respect to $x$. We assume:

(A1) The domain $\Omega$ has $C^{1,1}$-boundary $\Gamma$.

(A2) For any $h \in G$, the functions $\varphi(\cdot, h): \mathbb{R} \rightarrow \mathbb{R}, \psi(\cdot, \cdot, h): \mathbb{R} \times \mathbb{R} \rightarrow$ $\mathbb{R}$ and $b(\cdot, \cdot, h): \mathbb{R} \times \mathbb{R} \rightarrow \mathbb{R}$ are of class $C^{2}$. Moreover, for any fixed $u \in \mathbb{R}$ and $h \in G, b(\cdot, u, h): \mathbb{R} \rightarrow \mathbb{R}$ is monotonically 
decreasing. There is a bound $c_{G}>0$ such that

$$
|b(0,0, h)|+\left|D_{(y, u)} b(0,0, h)\right|+\left|D_{(y, u)}^{2} b(0,0, h)\right| \leq c_{G} \quad \forall h \in G .
$$

Moreover, for any $K>0$ there exists a constant $l(K)$ such that

$\left|D_{(y, u)}^{2} b\left(y_{1}, u_{1}, h\right)-D_{(y, u)}^{2} b\left(y_{2}, u_{2}, h\right)\right| \leq l(K)\left(\left|y_{1}-y_{2}\right|+\left|u_{1}-u_{2}\right|\right)$

for all $y_{i}, u_{i}$ such that $\left|y_{i}\right| \leq K,\left|u_{i}\right| \leq K$, and all $h \in G$. The same conditions as above are also satisfied by $\varphi$ and $\psi$.

(A3) The functions $b(y, u, \cdot), D_{y} b(y, u, \cdot)$ and $D_{u} b(y, u, \cdot)$ are Fréchet differentiable in $h$. Similar properties posses functions $\varphi$ and $\psi$.

By the following lemma, proved in [6], problem $\left(\mathrm{O}_{h}\right)$ is well posed.

Lemma 1 If (A1) - (A3) hold, then for any $u \in \mathcal{U}$ and any $h \in G$ there exists a unique weak solution $y(u, h) \in W^{1,2}(\Omega) \cap C(\bar{\Omega})$ of (3). Moreover, there exists $c>0$ such that

$$
\left\|y\left(u^{\prime}, h^{\prime}\right)-y\left(u^{\prime \prime}, h^{\prime \prime}\right)\right\|_{C(\bar{\Omega})} \leq c\left(\left\|u^{\prime}-u^{\prime \prime}\right\|_{L^{\infty}(\Gamma)}+\left\|h^{\prime}-h^{\prime \prime}\right\|_{H}\right) .
$$

Define the following Hamiltonian and Lagrangian

$$
\begin{aligned}
& \mathcal{H}: \mathbb{R}^{3} \times G \rightarrow \mathbb{R}, \quad \mathcal{L}: W^{1,2}(\Omega) \times L^{\infty}(\Gamma) \times W^{1,2}(\Omega) \times G \rightarrow \mathbb{R}, \\
& \mathcal{H}(y, u, p, h):=\psi(y, u, h)+p b(y, u, h) \\
& \mathcal{L}(y, u, p, h):=F(y, u, h)-\int_{\Omega} p(-\Delta y+y) d x \\
&=\int_{\Omega}[\varphi(y, h)-(\nabla p, \nabla y)-(p, y)] d x+\int_{\Gamma} \mathcal{H}(y, u, p, h) d S_{x} .
\end{aligned}
$$

We assume:

(A4) For a given reference value $h_{0} \in G$ of the parameter, there exists a local solution $\left(y_{0}, u_{0}\right) \in Z^{\infty}$ of $\left(\mathrm{O}_{h_{0}}\right)$ and an associated state $p_{0} \in W^{1,2}(\Omega) \cap C(\bar{\Omega})$, such that the following first-order necessary optimality conditions hold

$$
\begin{array}{cl}
D_{y} \mathcal{L}\left(y_{0}, u_{0}, p_{0}, h_{0}\right) z=0 & \text { for all } z \in W^{1,2}(\Omega), \\
D_{u} \mathcal{L}\left(y_{0}, u_{0}, p_{0}, h_{0}\right)\left(u-u_{0}\right) \geq 0 & \text { for all } u \in \mathcal{U} .
\end{array}
$$

In a standard way, conditions (8) and (9) yield the adjoint equation and the pointwise stationarity of the Hamiltonian:

$$
\left.\begin{array}{rlrl}
-\Delta p_{0}(x)+p_{0}(x) & =D_{y} \varphi\left(y_{0}(x), h_{0}\right), & & \text { in } \Omega, \\
\partial_{\nu} p_{0}(x) & =D_{y} \mathcal{H}\left(y_{0}(x), u_{0}(x), p_{0}(x), h_{0}\right), & & \text { on } \Gamma,
\end{array}\right\}
$$




$$
\left.\begin{array}{c}
D_{u} \mathcal{H}\left(y_{0}(x), u_{0}(x), p_{0}(x), h_{0}\right)\left(u-u_{0}(x)\right) \geq 0 \\
\text { for all } u \in\left[m_{1}, m_{2}\right] \text { and a.a. } x \in \Gamma .
\end{array}\right\}
$$

Conditions (10) and (11) together with the state equation (3) constitute the optimality system for $\left(\mathrm{O}_{h_{0}}\right)$. It will be convenient to rewrite this optimality system in the form of a generalized equation. To do that, define the spaces

$$
\begin{aligned}
& X^{s}:=W^{1, s}(\Omega) \times L^{s}(\Gamma) \times W^{1, s}(\Omega), \\
& \Delta^{s}=L^{s}(\Omega) \times L^{s}(\Gamma) \times L^{s}(\Omega) \times L^{s}(\Gamma) \times L^{s}(\Gamma), s \in[2, \infty]
\end{aligned}
$$

and the following set-valued mapping with closed graph:

$$
\mathcal{N}(u)= \begin{cases}\lambda \in\left\{L^{\infty}(\Gamma) \mid \int_{\Gamma} \lambda(v-u) d S_{x} \leq 0 \quad \forall v \in \mathcal{U}\right\} & \text { if } u \in \mathcal{U} \\ \emptyset & \text { if } u \notin \mathcal{U}\end{cases}
$$

Denote $\xi=(y, u, p) \in X^{\infty}$. Let the function $\mathcal{F}: X^{\infty} \times G \rightarrow \Delta^{\infty}$, as well as the multivalued mapping $\mathcal{T}: X^{\infty} \rightarrow 2^{\Delta^{\infty}}$ be defined as follows

$$
\mathcal{F}(\xi, h)=\left[\begin{array}{ll}
-\Delta y+y & \text { in } \Omega \\
\partial_{\nu} y-b(y, u, h) & \text { on } \Gamma \\
-\Delta p+p-D_{y} \varphi(y, h) & \text { in } \Omega \\
\partial_{\nu} p-D_{y} \mathcal{H}(y, u, p, h) & \text { on } \Gamma \\
D_{u} \mathcal{H}(y, u, p, h) & \text { on } \Gamma
\end{array}\right], \quad \mathcal{T}(\xi)=\left[\begin{array}{c}
\{0\} \\
\{0\} \\
\{0\} \\
\{0\} \\
\mathcal{N}(u)
\end{array}\right]
$$

Then the optimality system $(3),(10),(11)$ for $\left(\mathrm{O}_{h_{0}}\right)$ can be expressed in the form of the following generalized equation:

$$
0 \in \mathcal{F}\left(\xi_{0}, h_{0}\right)+\mathcal{T}\left(\xi_{0}\right) .
$$

\section{Application of abstract theorems for generalized equations}

We are going to investigate conditions under which there exists a neighborhood $G_{0}$ of $h_{0}$ such that, for each $h \in G_{0}$, the generalized equation

$$
0 \in \mathcal{F}(\xi, h)+\mathcal{T}(\xi)
$$

has a locally unique solution $\xi_{h}=\left(y_{h}, u_{h}, p_{h}\right)$, which is Bouligand differentiable function of $h$. We will follow the same scheme as in $[4,5]$. Namely the proof will be in two steps. First, we show existence, local uniqueness and Lipschitz continuity of the solutions to (16). In the 
second step, we use these properties, to show differentiability of the solutions. In both steps we need the following auxiliary generalized equation, obtained from (16) by linearization of $\mathcal{F}\left(\cdot, h_{0}\right)$ at the reference solution and by perturbation:

$$
\delta \in \mathcal{F}\left(\xi_{0}, h_{0}\right)+D_{\xi} \mathcal{F}\left(\xi_{0}, h_{0}\right)\left(\zeta-\xi_{0}\right)+\mathcal{T}(\zeta),
$$

where $\delta \in \Delta^{\infty}$ is the perturbation. Clearly, for $\delta=0, \xi_{0}$ is a solution to (17). We will denote by $\mathcal{B}_{\rho}^{X}\left(x_{0}\right):=\left\{x \in X \mid\left\|x-x_{0}\right\|_{X} \leq \rho\right\}$ the closed ball of radius $\rho$ centered at $x_{0}$ in a Banach space $X$.

The following Robinson's implicit function theorem (see, Theorem 2.1 and Corollary 2.2 in [7]) allows to deduce existence and local Lipschitz continuity of the solutions to the nonlinear generalized equation (16), from the same properties of the solutions to the linearized equation (17).

Theorem 1 If there exist $\rho_{1}>0$ and $\rho_{2}>0$ such that, for each $\delta \in$ $\mathcal{B}_{\rho_{1}}^{\Delta^{\infty}}(0)$ there is a unique solution $\zeta_{\delta}$ in $\mathcal{B}_{\rho_{2}}^{X}\left(\xi_{0}\right)$ of $(17)$, which is Lipschitz continuous in $\delta$, then there exist $\sigma_{1}>0$ and $\sigma_{2}>0$ such that, for each $h \in \mathcal{B}_{\sigma_{1}}^{H}\left(h_{0}\right)$ there is a unique solution $\xi_{h}$ in $\mathcal{B}_{\sigma_{2}}^{X}\left(\xi_{0}\right)$ of $(16)$, which is Lipschitz continuous in $h$.

Similarly, the following theorem due to Dontchev (see, Theorem 2.4 and Remark 2.6 in [2]) allows to reduce differentiability analysis for the solutions to (16) to the same analysis for the solutions to (17).

Theorem 2 If the assumptions of Theorem 1 are satisfied and, in addition, the solutions $\zeta_{\delta} \in \mathcal{B}_{\rho_{2}}^{X}\left(\xi_{0}\right)$ of (17) are Bouligand differentiable functions of $\delta$ in a neighborhood of the origin, with the differential $\left(D_{\delta} \zeta_{0} ; \eta\right)$, then the solutions $\xi_{h}$ of (16) are Bouligand differentiable in a neighborhood of $h_{0}$. For a direction $g \in H$, the differential at $h_{0}$ is given by

$$
\left(D_{h} \xi_{0} ; g\right)=\left(D_{\delta} \zeta_{0} ;-D_{h} \mathcal{F}\left(\xi_{0}, h_{0}\right) g\right)
$$

Remark 1 In Theorem 1, Lipschitz continuity of $\zeta$ and $\xi$ is understood in the sense of that norm in the space $X$, in which $\mathcal{F}(\cdot, h)$ is differentiable. On the other hand, Theorem 2 remains true, if B-differentiability of $\zeta_{\delta}$ is satisfied in a norm in the image space $X$ weaker than that in which Lipschitz continuity in Theorem 1 holds (see, Remark 2.11 in [2]); e.g., in $L^{s},(s<\infty)$, rather than in $L^{\infty}$. This property will be used in Section 4.

In order to apply Theorems 1 and 2 to $\left(\mathrm{O}_{h}\right)$, we have to find the form of the linearization (17) of the optimality system (16), for $\mathcal{F}$ and $\mathcal{T}$ given 
in (14). To simplify notation, the functions evaluated at the reference point will be denoted by subscript "0", e.g., $\varphi_{0}:=\varphi\left(y_{0}, h_{0}\right), \mathcal{H}_{0}:=$ $\mathcal{H}\left(y_{0}, u_{0}, p_{0}, h_{0}\right)$. Moreover, we denote $\xi_{0}:=\left(y_{0}, u_{0}, p_{0}\right)$.

Let $\delta=\left(\delta^{1}, \delta^{2}, \delta^{3}, \delta^{4}, \delta^{5}\right) \in \Delta^{\infty}$ be a vector of perturbations. By simple calculations we obtain the following form of (17):

$$
\begin{array}{ll}
\left.\left(\mathrm{LO}_{\delta}\right) \quad \begin{array}{l}
-\Delta z+z=e^{1}+\delta^{1} \\
\\
\partial_{\nu} z-D_{y} b_{0} z=e^{2}+\delta^{2}+D_{u} b_{0} v,
\end{array}\right\} \\
-\Delta q+q=e^{3}+\delta^{3}+D_{y y}^{2} \varphi_{0} z \\
\partial_{\nu} q-D_{y} b_{0} q=e^{4}+\delta^{4}+D_{y y}^{2} \mathcal{H}_{0} z+D_{y u}^{2} \mathcal{H}_{0} v, \\
& D_{u y}^{2} \mathcal{H}_{0} z+D_{u u}^{2} \mathcal{H}_{0} v+D_{u} b_{0} q-e^{5}-\delta^{5} \in-\mathcal{N}(v),
\end{array}
$$

where $e=\left(e^{1}, e^{2}, e^{3}, e^{4}, e^{5}\right) \in \Delta^{\infty}$ is a given vector.

Note that

$$
\left(z_{0}, v_{0}, q_{0}\right)=\left(y_{0}, u_{0}, p_{0}\right)
$$

is a solution to $\left(\mathrm{LO}_{\delta}\right)$ for $\delta=0$. An inspection shows that $\left(\mathrm{LO}_{\delta}\right)$ can be treated as an optimality system for the following linear-quadratic accessory problem:

$\left(\mathrm{LP}_{\delta}\right) \quad$ Find $\left(z_{\delta}, v_{\delta}\right) \in Z^{\infty}$ such that

$$
\mathcal{I}\left(z_{\delta}, v_{\delta}, \delta\right)=\min \mathcal{I}(z, v, \delta)
$$

subject to

$$
\begin{aligned}
& -\Delta z(x)+z(x)=\delta^{1}(x) \quad \text { in } \Omega, \\
& \partial_{\nu} z(x)=D_{y} b_{0}(x) z(x)+D_{u} b_{0}(x) v(x) \\
& +e^{2}(x)+\delta^{2}(x)
\end{aligned}
$$

$v \in \mathcal{U}$

where

$$
\begin{aligned}
\mathcal{I}(z, v, \delta) & :=\frac{1}{2}\left((z, v), D^{2} \mathcal{L}_{0}(z, v)\right)+\int_{\Omega}\left(e^{3}+\delta^{3}\right) z d x+ \\
& +\int_{\Gamma}\left[\left(e^{4}+\delta^{4}\right) z+\left(e^{5}+\delta^{5}\right) v\right] d S_{x}
\end{aligned}
$$

with the quadratic form

$$
\begin{aligned}
& \left((z, v), D^{2} \mathcal{L}_{0}(z, v)\right):=\int_{\Omega} D_{y y}^{2} \varphi\left(y_{0}, h_{0}\right) z^{2} d x \\
& \quad+\int_{\Gamma}[z, v]\left[\begin{array}{cc}
D_{y y}^{2} \mathcal{H}_{0} & D_{y u}^{2} \mathcal{H}_{0} \\
D_{u y}^{2} \mathcal{H}_{0} & D_{u u}^{2} \mathcal{H}_{0}
\end{array}\right]\left[\begin{array}{c}
z \\
v
\end{array}\right] d S_{x} .
\end{aligned}
$$

To verify assumptions of Theorems 1 and 2 , we have to show that there exist constants $\rho_{1}, \rho_{2}>0$ such that for each $\delta \in \mathcal{B}_{\rho_{1}}^{\Delta^{\infty}}(0)$ there is a unique stationary point $\zeta_{\delta}:=\left(z_{\delta}, v_{\delta}, q_{\delta}\right)$ in $\mathcal{B}_{\rho_{2}}^{X^{\infty}}\left(\xi_{0}\right)$ of $\left(\mathrm{LP}_{\delta}\right)$, which is a Lipschitz continuous and Bouligand differentiable function of $\delta$. 


\section{Differentiability of solutions to accessory problems}

As in [4], the starting point in the proof of differentiability of the solutions to $\left(\mathrm{LP}_{\delta}\right)$ is the Lipschitz continuity property for these solutions. To this end, we will need a coercivity assumption (see, [6]). Let us define the sets of those points, at which the reference control is active:

$$
I=\left\{x \in \Gamma \mid u_{0}(x)=m_{1}\right\}, \quad J=\left\{x \in \Gamma \mid u_{0}(x)=m_{2}\right\} .
$$

Moreover, for any $\alpha \geq 0$ define the sets

$$
\begin{aligned}
& I^{\alpha}=\left\{x \in \Gamma \mid D_{u} \mathcal{H}\left(y_{0}, u_{0}, p_{0}, h_{0}\right)(x)>\alpha\right\}, \\
& J^{\alpha}=\left\{x \in \Gamma \mid-D_{u} \mathcal{H}\left(y_{0}, u_{0}, p_{0}, h_{0}\right)(x)>\alpha\right\} .
\end{aligned}
$$

As in [6], we assume:

(AC) (coercivity) There exist $\alpha>0$ and $\gamma>0$ such that

$$
\left((z, v), D^{2} \mathcal{L}_{0}(z, v)\right) \geq \gamma\|v\|_{L^{2}(\Gamma)}^{2}
$$

for all pairs $(z, v)$ satisfying

$$
\begin{array}{ll}
-\Delta z(x)+z(x)=0 & \text { in } \Omega, \\
\partial_{\nu} z(x)-D_{y} b_{0}(x) z(x)-D_{u} b_{0}(x) v(x)=0 & \text { in } \Gamma,
\end{array}
$$

and such that $v \in\left\{L^{2}(\Gamma) \mid v(x)=0\right.$ for a.a. $\left.x \in I^{\alpha} \cup J^{\alpha}\right\}$.

Note that (AC) implies the following pointwise coercivity condition (see, e.g., Lemma 5.1 in [10]).

$$
D_{u u}^{2} \mathcal{H}_{0}(x) \geq \gamma \quad \text { for a.a. } x \in \Gamma \backslash\left(I^{\alpha} \cup J^{\alpha}\right) .
$$

By a slight modification of Satz 18 in [11] we get the following Lipschitz continuity result for $\left(\mathrm{LP}_{\delta}\right)$ :

Proposition 1 If (AC) holds, then there exist constants $\rho_{1}>0$ and $\rho_{2}>0$ such that, for all $\delta \in \mathcal{B}_{\rho_{1}}^{\Delta^{\infty}}(0)$ there is a unique stationary point $\zeta_{\delta}:=\left(z_{\delta}, v_{\delta}, q_{\delta}\right)$ in $\mathcal{B}_{\rho_{2}}^{X^{\infty}}\left(\xi_{0}\right)$ of $\left(\mathrm{LP}_{\delta}\right)$. Moreover, there exists a constant $\ell>0$ such that

$\left\|z_{\delta^{\prime}}-z_{\delta^{\prime \prime}}\right\|_{W^{1, s}(\Omega)},\left\|v_{\delta^{\prime}}-v_{\delta^{\prime \prime}}\right\|_{L^{s}(\Gamma)},\left\|q_{\delta^{\prime}}-q_{\delta^{\prime \prime}}\right\|_{W^{1, s}(\Omega)}, \leq \ell\left\|\delta^{\prime}-\delta^{\prime \prime}\right\|_{\Delta^{s}}$ for all $\delta^{\prime}, \delta^{\prime \prime} \in \mathcal{B}_{\rho_{1}}^{\Delta^{\infty}}(0)$ and all $s \in[2, \infty]$ 
The proof of B-differentiability of the stationary points of $\left(\mathrm{LP}_{\delta}\right)$ is in two steps. In the first step, directional differentiability is proved and the directional differential is characterized. This characterization is used in the second step to show that the differential is actually Bouligand. Let us start with the directional differentiability. The proof of the following result is very similar to that of Proposition 4.3 in [5].

Proposition 2 Let (A1)-(A3) as well as (AC) be satisfied and let $\rho_{1}, \rho_{2}>0$ be as in Proposition 1. Then the mapping

$$
\zeta_{\delta}:=\left(z_{\delta}, v_{\delta}, q_{\delta}\right): \mathcal{B}_{\rho_{1}}^{\Delta^{\infty}}(0) \rightarrow X^{2},
$$

where $\zeta_{\delta} \in \mathcal{B}_{\rho_{2}}^{X^{\infty}}\left(\xi_{0}\right)$ denote a unique stationary point of $\left(\mathrm{LP}_{\delta}\right)$, is directionally differentiable. The directional differential at $\delta=0$, in a direction $\eta \in \Delta^{\infty}$, is given by $\left(\varpi_{\eta}, w_{\eta}, r_{\eta}\right)$, where $\left(\varpi_{\eta}, w_{\eta}\right)$ is the solution and $r_{\eta}$ the associated adjoint state of the following linear-quadratic optimal control problem:

$$
\begin{aligned}
& \left(\mathrm{LQ}_{\eta}\right) \quad \text { Find }\left(\varpi_{\eta}, w_{\eta}\right) \in W^{1,2}(\Omega) \times L^{2}(\Gamma) \text { that minimizes } \\
& \mathcal{J}_{\eta}(\varpi, w)=\frac{1}{2}\left((\varpi, w), D^{2} \mathcal{L}_{0}(\varpi, w)\right)+\int_{\Omega} \eta^{3} \varpi d x \\
& +\int_{\Gamma}\left(\eta^{4} \varpi+\eta^{5} w\right) d S_{x} \\
& \text { subject to } \\
& \begin{aligned}
-\Delta \varpi+\varpi & =\eta^{1} & & \text { in } \Omega, \\
\partial_{\nu} \varpi & =D_{y} b_{0} \varpi+D_{u} b_{0} w+\eta^{2} & & \text { on } \Gamma,
\end{aligned} \\
& w(x) \begin{cases}=0 & \text { for } x \in\left(I^{0} \cup J^{0}\right), \\
\geq 0 & \text { for } x \in\left(I \backslash I^{0}\right), \\
\leq 0 & \text { for } x \in\left(J \backslash J^{0}\right), \\
\text { free } & \text { for } x \in \Gamma \backslash(I \cup J) .\end{cases}
\end{aligned}
$$

Note that, by the same argument as in Proposition 1, we find that the stationary points of $\left(\mathrm{LQ}_{\eta}\right)$ are Lipschitz continuous functions of $\eta$. Since $\left(\varpi_{0}, w_{0}, r_{0}\right)=(0,0,0)$, we have

$$
\left\|\varpi_{\eta}\right\|_{W^{1, s}(\Omega)},\left\|w_{\eta}\right\|_{L^{s}(\Gamma)},\left\|r_{\eta}\right\|_{W^{1, s}(\Omega)} \leq \ell\|\eta\|_{\Delta^{s}}, \quad s \in[2, \infty] .
$$

We are now going to show that $\left(\varpi_{\eta}, w_{\eta}\right)$ and $r_{\eta}$ are actually Bdifferentials at $\delta=0$ of $\left(z_{\delta}, v_{\delta}\right)$ and $q_{\delta}$, respectively.

Theorem 3 Let (A1)-(A3) as well as (AC) be satisfied and let $\rho_{1}, \rho_{2}>$ 0 be as in Proposition 1. Then the mapping

$$
\zeta_{\delta}:=\left(z_{\delta}, v_{\delta}, q_{\delta}\right): \mathcal{B}_{\rho_{1}}^{\Delta^{\infty}}(0) \rightarrow X^{s}
$$


where $\zeta_{\delta} \in \mathcal{B}_{\rho_{2}}^{X^{\infty}}\left(\xi_{0}\right)$ denote a unique stationary point of $\left(\mathrm{LP}_{\delta}\right)$, is $B$ differentiable for any $s \in[2, \infty)$. The $B$-differential at $\delta=0$ in a direction $\eta \in \Delta$ is given by $\vartheta_{\eta}:=\left(\varpi_{\eta}, w_{\eta}, r_{\eta}\right)$, where $\left(\varpi_{\eta}, w_{\eta}\right)$ is the solution and $r_{\eta}$ the associated adjoint state of problem $\left(\mathrm{LQ}_{\eta}\right)$.

Proof The optimality system for $\left(\mathrm{LQ}_{\eta}\right)$ takes the form:

$$
\begin{aligned}
& \left.\begin{array}{l}
-\Delta \varpi+\varpi=\eta^{1} \\
\partial_{\nu} \varpi-D_{y} b_{0} \varpi=\eta^{2}+D_{u} b_{0} w,
\end{array}\right\} \\
& \left.\begin{array}{l}
-\Delta r+r=\eta^{3}+D_{y y}^{2} \varphi_{0} \varpi, \\
\partial_{\nu} r-D_{y} b_{0} r=\eta^{4}+D_{y y}^{2} \mathcal{H}_{0} \varpi+D_{y u}^{2} \mathcal{H}_{0} w .
\end{array}\right\} \\
& \left.\begin{array}{l}
\left(D_{u y}^{2} \mathcal{H}_{0} \varpi+D_{u u}^{2} \mathcal{H}_{0} w-D_{u} b_{0} r-\eta^{5}, v-w\right) \geq 0 \\
\text { for all } v \in L^{2}(Q) \text { satisfying }(32) .
\end{array}\right\}
\end{aligned}
$$

We have to show that the solution $\left(\varpi_{\eta}, w_{\eta}, r_{\eta}\right)$ of $(35)-(37)$ are Bdifferentials of the solution to $\left(\mathrm{LO}_{\delta}\right)$. Clearly, $\left(\varpi_{\eta}, w_{\eta}, r_{\eta}\right)$ is a positively homogeneous function of $\eta$, so, by Definition 1, it is enough to show that

$$
\begin{aligned}
& z_{\eta}=z_{0}+\varpi_{\eta}+\sigma_{1}(\eta), \quad v_{\eta}=v_{0}+w_{\eta}+\sigma_{2}(\eta), \quad q_{\eta}=q_{0}+r_{\eta}+\sigma_{1}(\eta), \\
& \text { where } \frac{\left\|\sigma_{1}(\eta)\right\|_{W^{1, s}(\Omega)}}{\|\eta\|_{\Delta^{\infty}}} \rightarrow 0, \quad \frac{\left\|\sigma_{2}(\eta)\right\|_{L^{s}(Q)}}{\|\eta\|_{\Delta^{\infty}}} \rightarrow 0, \quad \text { as }\|\eta\|_{\Delta^{\infty}} \rightarrow 0, \\
& \text { for any } s \in[2, \infty) .
\end{aligned}
$$

Denote

$$
\left(z_{\eta}-z_{0}\right)=\widetilde{\varpi}_{\eta}, \quad\left(v_{\eta}-v_{0}\right)=\widetilde{w}_{\eta}, \quad\left(q_{\eta}-q_{0}\right)=\widetilde{r}_{\eta} .
$$

It follows from (19) and (20) that $\left(\widetilde{\varpi}_{\eta}, \widetilde{w}_{\eta}, \widetilde{r}_{\eta}\right)$ satisfies equations identical with (35) and (36):

$$
\left.\begin{array}{l}
-\Delta \widetilde{\varpi}+\widetilde{\varpi}=\eta^{1}, \\
\partial_{\nu} \widetilde{\varpi}-D_{y} b_{0} \widetilde{\varpi}=\eta^{2}+D_{u} b_{0} w,
\end{array}\right\}
$$

To characterize $\left(\widetilde{\varpi}_{\eta}, \widetilde{w}_{\eta}, \widetilde{r}_{\eta}\right)$, we still need a condition analogous to (37). To this end, let us choose $\beta \in(0, \alpha)$, where $\alpha$ is given in (AC). Define the sets

$$
\begin{aligned}
& K_{1}^{\beta}=\left\{x \in I^{0} \mid D_{u u}^{2} \mathcal{H}_{0}(x) \in(0, \beta)\right\} \\
& K_{2}^{\beta}=\left\{x \in J^{0} \mid-D_{u u}^{2} \mathcal{H}_{0}(x) \in(0, \beta)\right\}, \\
& L^{\beta}=\left\{x \in \Gamma \mid u_{0}(x) \in\left(m_{1}, m_{1}+\beta\right) \cup\left(m_{2}-\beta, m_{2}\right)\right\} .
\end{aligned}
$$


Note that

$$
\text { meas }\left(K_{1}^{\beta} \cup K_{2}^{\beta} \cup L^{\beta}\right) \rightarrow 0 \text { as } \beta \rightarrow 0 .
$$

Let us split up the set $\Gamma$ into the following subsets

$$
\begin{array}{ll}
\mathcal{A}=\Gamma \backslash\left(I \cup J \cup L^{\beta}\right), & \mathcal{B}=\left(I^{0} \backslash K_{1}^{\beta}\right) \cup\left(J^{0} \backslash K_{2}^{\beta}\right), \\
\mathcal{C}=\left(I \backslash I^{0}\right) \cup\left(J \backslash J^{0}\right), & \mathcal{D}=K_{1}^{\beta} \cup K_{2}^{\beta} \cup L^{\beta} .
\end{array}
$$

We will analyze conditions analogous to (37) on each of these subsets successively.

Subset $\mathcal{A}$ Choose $\varrho(\beta)=\ell^{-1} \beta$. Then by (22) and (25), as well as by Proposition 1, for all $\eta \in \mathcal{B}_{\varrho(\beta)}^{\Delta \infty}(0)$ we get

$$
v_{\eta}(x) \in\left(m_{1}, m_{2}\right) \quad \text { for a.a. } x \in \mathcal{A},
$$

i.e., by $(21)$

$$
\begin{gathered}
D_{u y}^{2} \mathcal{H}_{0}(x) z_{\eta}(x)+D_{u u}^{2} \mathcal{H}_{0}(x) v_{\eta}(x)+D_{u} b_{0}(x, t) q_{\eta}(x, t) \\
-e^{5}(x)-\eta^{5}(x)=0 \quad \text { for a.a. } x \in \mathcal{A} .
\end{gathered}
$$

Subtracting from (45) the analogous equation for $\left(z_{0}, v_{0}, q_{0}\right)$ and using notation (39), we obtain

$$
\begin{gathered}
D_{u y}^{2} \mathcal{H}_{0}(x) \widetilde{\varpi}_{\eta}(x)+D_{u u}^{2} \mathcal{H}_{0}(x) \widetilde{w}_{\eta}(x)-D_{u} a_{0}(x) \widetilde{r}_{\eta}(x) \\
-\eta^{5}(x)=0 \quad \text { for a.a. } x \in \mathcal{A} .
\end{gathered}
$$

Subset $\mathcal{B}$ It follows from Proposition 1 that, shrinking $\varrho(\beta)>0$ if necessary, for all $\eta \in \mathcal{B}_{\varrho(\beta)}^{\Delta \infty}(0)$ we obtain

$$
\begin{aligned}
& D_{u y}^{2} \mathcal{H}_{0}(x) z_{\eta}(x)+D_{u u}^{2} \mathcal{H}_{0}(x) v_{\eta}(x)+D_{u} b_{0}(x) q_{\eta}(x) \\
& -e^{5}(x)-\eta^{5}(x) \begin{cases}>0 & \text { for a.a. } x \in I^{0} \backslash K_{1}^{\beta}, \\
<0 & \text { for a.a. } x \in J^{0} \backslash K_{2}^{\beta},\end{cases}
\end{aligned}
$$

which, by (21) implies

$$
v_{\eta}(x)= \begin{cases}m_{1}(x) & \text { for a.a. } x \in I^{0} \backslash K_{1}^{\beta}, \\ m_{2}(x) & \text { for a.a. } x \in J^{0} \backslash K_{2}^{\beta},\end{cases}
$$

i.e.,

$$
\widetilde{w}_{\eta}(x)=0 \quad \text { for a.a. } x \in \mathcal{B} .
$$

$\underline{\text { Subset } \mathcal{C}}$ By (22) and (25) we have

$$
v_{0}(x)=u_{0}(x)= \begin{cases}m_{1}(x) & \text { for a.a. } x \in I \backslash I^{0}, \\ m_{2}(x) & \text { for a.a. } x \in J \backslash J^{0}\end{cases}
$$


and

$$
\begin{aligned}
& D_{u y}^{2} \mathcal{H}_{0}(x) z_{0}(x)+D_{u u}^{2} \mathcal{H}_{0}(x) v_{0}(x)+D_{u} b_{0}(x) q_{0}(x)=0 \\
& \text { for a.a. } x \in\left(I \backslash I^{0}\right) \cup\left(J \backslash J^{0}\right) .
\end{aligned}
$$

Proposition 1, together with (49) implies that, shrinking $\varrho(\beta)$ if necessary, for any $\eta \in \mathcal{B}_{\varrho(\beta)}^{\Delta^{\infty}}(0)$ we get

$$
v_{\eta}(x) \in \begin{cases}{\left[m_{1}, m_{2}\right)} & \text { for a.a. } x \in I \backslash I^{0}, \\ \left(m_{1}, m_{2}\right] & \text { for a.a. } x \in J \backslash J^{0} .\end{cases}
$$

Hence, in view of (21) we have

$$
\begin{gathered}
D_{u y}^{2} \mathcal{H}_{0}(x) z_{\eta}(x)+D_{u u}^{2} \mathcal{H}_{0}(x) v_{\eta}(x)+D_{u} b_{0}(x) q_{\eta}(x) \\
-\eta^{5}(x) \begin{cases}\geq 0 & \text { for a.a. } x \in I \backslash I^{0}, \\
\leq 0 & \text { for a.a. } x \in J \backslash J^{0} .\end{cases}
\end{gathered}
$$

Conditions (49)-(52) imply:

$$
\begin{aligned}
& \widetilde{w}_{\eta}(x) \begin{cases}\geq 0 & \text { for a.a. } x \in I \backslash I^{0}, \\
\leq 0 & \text { for a.a. } x \in J \backslash J^{0}\end{cases} \\
& D_{u y}^{2} \mathcal{H}_{0}(x) \widetilde{\varpi}_{\eta}(x)+D_{u u}^{2} \mathcal{H}_{0}(x) \widetilde{w}_{\eta}(x)+D_{u} b_{0}(x) \widetilde{r}_{\eta}(x) \\
& -\eta^{5}(x) \begin{cases}\geq 0 & \text { for a.a. } x \in I \backslash I^{0}, \\
\leq 0 & \text { for a.a. } x \in J \backslash J^{0},\end{cases}
\end{aligned}
$$

and

$$
\begin{aligned}
& \left(D_{u y}^{2} \mathcal{H}_{0}(x) \widetilde{\varpi}_{\eta}(x)+D_{u u}^{2} \mathcal{H}_{0}(x) \widetilde{w}_{\eta}(x)+D_{u} b_{0}(x) \widetilde{r}_{\eta}(x)\right. \\
& \left.\quad-\eta^{3}(x)\right)\left(w-\widetilde{w}_{\eta}(x)\right) \geq 0 \begin{cases}\text { for all } w \geq 0 & \text { on } I \backslash I^{0} \\
\text { for all } w \leq 0 & \text { on } J \backslash J^{0}\end{cases}
\end{aligned}
$$

Subset $\mathcal{D}$ The analysis of subset $\mathcal{D}$ is the most difficult, because we do not know a priori if for $x \in \mathcal{D}$ the constraints are active or not at $v_{\eta}$, no matter how small $\eta$ is chosen. Without this information, we can say very few about $\widetilde{w}_{\eta}(x)=v_{\eta}(x)-v_{0}(x)$. Let us denote

$$
\begin{gathered}
\left(\widetilde{\eta}^{5}\right)^{\prime}(x)=D_{u y}^{2} \mathcal{H}_{0}(x)\left(z_{\eta}(x)-z_{0}(x)\right)+D_{u u}^{2} \mathcal{H}_{0}(x)\left(v_{\eta}(x)-v_{0}(x)\right) \\
+D_{u} b_{0}(x)\left(q_{\eta}(x)-q_{0}(x)\right) \text { for a.a. } x \in \mathcal{D}
\end{gathered}
$$

By definition (39) we have

$$
\begin{aligned}
& D_{u y}^{2} \mathcal{H}_{0}(x) \widetilde{\varpi}_{\eta}(x)+D_{u u}^{2} \mathcal{H}_{0}(x) \widetilde{v}_{\eta}(x)+D_{u} b_{0}(x) \widetilde{r}_{\eta}(x) \\
& -\left(\widetilde{\eta}^{5}\right)^{\prime}(x)=0 \quad \text { for a.a. } x \in \mathcal{D} .
\end{aligned}
$$


Denote $\eta^{\prime}=\left(\eta^{1}, \eta^{2}, \eta^{3}, \eta^{4},\left(\eta^{5}\right)^{\prime}\right)$, where

$$
\left(\eta^{5}\right)^{\prime}(x)= \begin{cases}\left(\widetilde{\eta}^{5}\right)^{\prime}(x) & \text { for } x \in \mathcal{D} \\ \eta^{5}(x) & \text { otherwise }\end{cases}
$$

It is easy to see that (40) and (41) together with (46), (48), (53)-(55) and (57) can be interpreted as an optimality system for the optimal control problem $\left(\widetilde{\mathrm{LQ}}_{\eta^{\prime}}\right)$, where $\left(\widetilde{\mathrm{LQ}}_{\eta}\right)$ is the following slight modification of $\left(\mathrm{LQ}_{\eta}\right)$ :

$\left(\widetilde{\mathrm{LQ}}{ }_{\eta}\right)$ Find $\left(\widetilde{\varpi}_{\eta}, \widetilde{w}_{\eta}\right) \in W^{2} \times L^{2}(\Gamma)$ that minimizes

$$
\begin{aligned}
& \mathcal{F}_{\eta}(\varpi, w) \quad \text { subject to } \\
& -\Delta \varpi(x)+\varpi(x)=\eta^{1}(x) \quad \text { in } \Omega, \\
& \partial_{\nu} \varpi+D_{y} b_{0} \varpi=D_{y} b_{0}(x) \varpi(x)+D_{u} b_{0}(x) w(x)+\eta^{2} \text { on } \Gamma, \\
& w(x) \begin{cases}=0 & \text { for } x \in\left(I^{0} \backslash K_{1}^{\beta}\right) \cup\left(J^{0} \backslash K_{2}^{\beta}\right), \\
\geq 0 & \text { for } x \in\left(I \backslash I^{0}\right), \\
\leq 0 & \text { for } x \in\left(J \backslash J^{0}\right), \\
\text { free } & \text { for } x \in \Gamma \backslash(I \cup J)) \cup\left(K_{1}^{\beta} \cup K_{2}^{\beta}\right) .\end{cases}
\end{aligned}
$$

Similarly $\left(\varpi_{\eta}, w_{\eta}, r_{\eta}\right)$ can be interpreted as a stationary point of $\left(\widetilde{\mathrm{LQ}}_{\eta^{\prime \prime}}\right)$, where $\eta^{\prime \prime}=\left(\eta^{1}, \eta^{2}, \eta^{3}, \eta^{4},\left(\eta^{5}\right)^{\prime \prime}\right)$, with

$$
\begin{aligned}
& \left(\eta^{5}\right)^{\prime \prime}(x)= \begin{cases}\left(\widetilde{\eta}^{5}\right)^{\prime \prime}(x) & \text { for } x \in \mathcal{D}, \\
\eta^{5}(x) & \text { otherwise, }\end{cases} \\
& \left(\widetilde{\eta}^{5}\right)^{\prime \prime}(x)=D_{u y}^{2} \mathcal{H}_{0}(x) \varpi_{\eta}(x)+D_{u u}^{2} \mathcal{H}_{0}(x) w_{\eta}(x)+D_{u} b_{0}(x) r_{\eta}(x)
\end{aligned}
$$

It can be easily checked that, as in the case of $\left(L Q_{\eta}\right)$, the stationary points of $\left(\widetilde{\mathrm{LQ}}_{\eta}\right)$ are Lipschitz continuous functions of $\eta$. Hence, in view of (58) and (59), we have

$$
\begin{aligned}
& \left\|\widetilde{\varpi}_{\eta}-\varpi_{\eta}\right\|_{W^{1, s}(\Omega)},\left\|\widetilde{w}_{\eta}-w_{\eta}\right\|_{L^{s}(\Gamma)},\left\|\widetilde{r}_{\eta}-r_{\eta}\right\|_{W^{1, s}(\Omega)} \\
& \quad \leq \ell\left\|\eta^{\prime}-\eta^{\prime \prime}\right\|_{\Delta^{s}}=\ell\left\{\int_{K_{1}^{\beta} \cup K_{2}^{\beta} \cup L^{\beta}}\left|\left(\widetilde{\eta}^{5}\right)^{\prime}(x)-\left(\widetilde{\eta}^{5}\right)^{\prime \prime}(x)\right|^{s} d S_{x}\right\}^{\frac{1}{s}}
\end{aligned}
$$


Using the definitions (56), (59) and taking advantage of (29) and of (33) we get

$$
\begin{aligned}
& \left|\left(\widetilde{\eta}^{5}\right)^{\prime}(x)-\left(\widetilde{\eta}^{5}\right)^{\prime \prime}(x)\right| \leq\left|\left(\widetilde{\eta}^{5}\right)^{\prime}(x)\right|+\left|\left(\widetilde{\eta}^{5}\right)^{\prime \prime}(x)\right| \\
& =\mid D_{u y}^{2} \mathcal{H}_{0}(x)\left(z_{\eta}(x)-z_{0}(x)\right)+D_{u u}^{2} \mathcal{H}_{0}(x)\left(v_{\eta}(x)-v_{0}(x)\right) \\
& \quad+D_{u} b_{0}(x)\left(q_{\eta}(x)-q_{0}(x)\right) \mid \\
& \quad+\left|D_{u y}^{2} \mathcal{H}_{0}(x) \varpi_{\eta}(x)+D_{u u}^{2} \mathcal{H}_{0}(x) v_{\eta}(x)+D_{u} b_{0}(x) r_{\eta}(x)\right| \\
& \quad \leq c\|\eta\|_{\Delta^{\infty}} \quad \text { for a.a. } x \in K_{1}^{\beta} \cup K_{2}^{\beta} \cup L^{\beta} .
\end{aligned}
$$

Substituting (61) to (60) we obtain

$$
\begin{gathered}
\left\|\widetilde{\varpi}_{\eta}-\varpi_{\eta}\right\|_{W^{1, s}(\Omega)},\left\|\widetilde{w}_{\eta}-w_{\eta}\right\|_{L^{s}(\Gamma)},\left\|\widetilde{r}_{\eta}-r_{\eta}\right\|_{W^{1, s}(\Omega)} \\
\leq c\|\eta\|_{\Delta^{\infty}}\left\{\operatorname{meas}\left(K_{1}^{\beta} \cup K_{2}^{\beta} \cup L^{\beta}\right)\right\}^{\frac{1}{s}} .
\end{gathered}
$$

In view of (39) and(43), we find that for any $\epsilon>0$ and any $s \in[2, \infty)$ we can choose $\beta(\epsilon, s)>0$ and the corresponding $\varrho(\beta(\epsilon, s))$, so small that

$$
\begin{gathered}
\left\|z_{\eta}-z_{0}-\varpi_{\eta}\right\|_{W^{1, s}(\Omega)},\left\|v_{\eta}-v_{0}-w_{\eta}\right\|_{L^{s}(\Gamma)},\left\|q_{\eta}-q_{0}-r_{\eta}\right\|_{W^{1, s}(\Omega)} \\
\leq \epsilon\|\eta\|_{\Delta^{\infty}} \text { for all } \eta \in \mathcal{B}_{\varrho(\beta(\epsilon, s))}^{\Delta^{\infty}}(0) .
\end{gathered}
$$

This shows that (38) holds and completes the proof of the theorem.

Remark 2 The proof of Theorem 3 cannot be repeated for $s=\infty$ and the counterexample in [4] shows that B-differentiability of (34) cannot be expected for $s=\infty$.

\section{Differentiability of the solutions to nonlinear problems}

By Theorems 2 and 3, for any $h$ in a neighborhood of $h_{0},\left(\mathrm{O}_{h}\right)$ has a unique stationary point $\left(y_{h}, u_{h}, p_{h}\right)$, which is a B-differentiable function of $h$. On the other hand, by Theorem 3.7 in [6], for $h$ sufficiently close to $h_{0}$, condition (AC) implies that $\left(y_{\delta}, u_{\delta}\right)$ is a solution to $\left(\mathrm{O}_{h}\right)$. Thus, we obtain the following principal result of this paper:

Theorem 4 If (A1)-(A7) and (AC) hold, then there exist constants $\sigma_{1}, \sigma_{2}>0$ such that, for any $h \in \mathcal{B}_{\sigma_{1}}^{H}\left(h_{0}\right)$, there is a unique stationary point $\left(y_{h}, u_{h}, p_{h}\right)$ in $\mathcal{B}_{\sigma_{2}}^{X^{\infty}}\left(\xi_{0}\right)$ of $\left(\mathrm{O}_{h}\right)$, where $\left(y_{h}, u_{h}\right)$ is a solution of $\left(\mathrm{O}_{h}\right)$. The mapping

$$
\left(y_{h}, u_{h}, p_{h}\right): \mathcal{B}_{\sigma_{1}}^{H}\left(h_{0}\right) \rightarrow X^{s}, \quad s \in[2, \infty)
$$


is B-differentiable, and the B-differential evaluated at $h_{0}$ in a direction $g \in H$ is given by the solution and adjoint state of the following linearquadratic optimal control problem

$$
\begin{aligned}
& \left(\mathrm{L}_{g}\right) \quad \text { Find }\left(z_{g}, v_{g}\right) \in W^{2} \times L^{2}(\Gamma) \text { that minimizes } \\
& \qquad \begin{array}{l}
\mathcal{K}_{g}(z, v)=\frac{1}{2}\left((z, v), D^{2} \mathcal{L}_{0}(z, v)\right)+\int_{\Omega} D_{y h}^{2} \varphi_{0} g z d x \\
+\int_{\Gamma} D_{y h}^{2} \mathcal{H}_{0} g z d S_{x}+\int_{\Gamma} D_{u h}^{2} \mathcal{H}_{0} g v d S_{x}
\end{array}
\end{aligned}
$$

subject to

$$
\begin{aligned}
-\Delta z+z & =0 & & \text { in } \Omega, \\
\partial_{\nu} z & =D_{y} b_{0} z+D_{u} b_{0} v+D_{h} b_{0} g & & \text { on } \Gamma,
\end{aligned}
$$

and

$$
v(x) \begin{cases}=0 & \text { for } x \in\left(I^{0} \cup J^{0}\right), \\ \geq 0 & \text { for } x \in\left(I \backslash I^{0}\right), \\ \leq 0 & \text { for } x \in\left(J \backslash J^{0}\right), \\ \text { free } & \text { for } x \in \Gamma \backslash(I \cup J)) .\end{cases}
$$

As it was noticed in Introduction, Bouligand differential becomes Fréchet if it is linear. Hence from the form of $\left(\mathrm{L}_{g}\right)$, we obtain immediately:

Corollary 1 If meas $\left(I \backslash I^{0}\right)=$ meas $\left(J \backslash J^{0}\right)=0$, then the mapping (63) is Fréchet differentiable.

In sensitivity analysis of optimization problems an important role is played by the so-called optimal value function, which on $\mathcal{B}_{\sigma_{1}}^{H}\left(h_{0}\right)$ is defined by:

$$
\mathcal{F}^{0}(h):=\mathcal{F}_{h}\left(y_{h}, u_{h}\right),
$$

i.e., to each $h \in \mathcal{B}_{\sigma_{1}}^{H}\left(h_{0}\right), \mathcal{F}^{0}$ assigns the (local) optimal value of the cost functional. In exactly the same way as in Corollary 5.3 in [5], we obtain the following result showing that Bouligand differentiability of the solutions implies the second order expansion of $\mathcal{F}_{0}$, uniform in a neighborhood of $h_{0}$.

Corollary 2 If assumptions of Theorem 4 hold, then for each $h=h_{0}+$ $g \in \mathcal{B}_{\sigma_{1}}^{H}\left(h_{0}\right)$

$$
\begin{aligned}
\mathcal{F}^{0}(h) & =\mathcal{F}^{0}\left(h_{0}\right)+\left(D_{h} \mathcal{L}_{0}, g\right) \\
& +\frac{1}{2}\left(\left(z_{g}, v_{g}, g\right),\left(\begin{array}{ccc}
D_{y y}^{2} \mathcal{L}_{0} & D_{y u}^{2} \mathcal{L}_{0} & D_{y h}^{2} \mathcal{L}_{0} \\
D_{u y}^{2} \mathcal{L}_{0} & D_{u u}^{2} \mathcal{L}_{0} & D_{u h}^{2} \mathcal{L}_{0} \\
D_{h y}^{2} \mathcal{L}_{0} & D_{h u}^{2} \mathcal{L}_{0} & D_{h h}^{2} \mathcal{L}_{0}
\end{array}\right)\left(z_{g}, v_{g}, g\right)\right) \\
& +o\left(\|g\|_{H}^{2}\right),
\end{aligned}
$$


where $\left(z_{g}, v_{g}\right)$ is the B-differential of $\left(y_{h}, u_{h}\right)$ at $h_{0}$ in the direction $g$, i.e., it is given by the solution to $\left(\mathrm{L}_{g}\right)$.

\section{References}

[1] Bonnans, J.F. (1998). "Second order analysis for control constrained optimal control problems of semilinear elliptic systems", Appl. Math. Optim., 38, 303325 .

[2] Dontchev, A.L. (1995). "Characterization of Lipschitz stability in optimization", In: R.Lucchetti, J.Revalski eds., Recent Developments in Well-Posed Variational Problems, Kluwer, pp. 95-116.

[3] Dontchev A.L. (1995). "Implicit function theorems for generalized equations", Math. Program., 70, 91-106.

[4] Malanowski, K. (2001). "Bouligand differentiability of solutions to parametric optimal control problems", Num. Funct. Anal. and Optim, 22, 973-990.

[5] Malanowski, K. (2002). "Sensitivity analysis for parametric optimal control of semilinear parabolic equations", J.Convex Anal., 9, 543-561.

[6] Malanowski, K. and Tröltzsch, F. (2000). "Lipschitz stability of solutions to parametric optimal control problems for elliptic equations", Control Cybern., 29, 237256.

[7] Robinson, S.M. (1980). "Strongly regular generalized equations", Math. Oper. Res., 5, 43-62.

[8] Robinson, S.M. (1987). "Local structure of feasible sets in nonlinear programming, Part III: Stability and sensitivity", Math. Program. Study, 30, 97-116.

[9] Shapiro, A. (1990). "On concepts of directional differentiability", J. Math. Anal. Appl., 66, 477-487.

[10] Tröltzsch, F. (2000). "Lipschitz stability of solutions to linear-quadratic parabolic control problems with respect to perturbations", Discr. Cont. Dynam. Systems 6, 289-306.

[11] Unger, A. (1997). Hinreichende Optimalitätsbedingungen 2. Ordnung und Konvergenz des SQP-Verfahrens für semilineare elliptische Randsteuerprobleme. $\mathrm{Ph}$. D. Thesis, Technische Universität Chemnitz-Zwickau. 